\title{
COMUNICACIÓN
}

\section{Patrones de actividad de hormigas urbanas y su preferencia de cebos en Alajuela, Costa Rica}

\author{
Pablo R. Gutiérrez-Martínez
}

Escuela de Biología, Universidad de Costa Rica, San José, Costa Rica; pablorgmbiol@hotmail.com

Recibido 28-XI-2017 • Corregido 05-XII-2017 • Aceptado 11-XII-2017

\begin{abstract}
Activity patterns of urban ants and their bait preference in Alajuela, Costa Rica. Ants have specific feeding preferences and daily activity cycles, but there are almost no data on tropical urban species. Through field collection and application of baits in Alajuela, Costa Rica, I obtained 902 records. I identified 15 urban ant species, with preference for certain foods, and daily cycles affected by food availability and the presence of dominant species.
\end{abstract}

Key words: circadian cycle, glucose, fructose, sucrose, chrono-biology, photoperiod, "extirpator" ants.
Resumen: Las hormigas tienen preferencias específicas de alimentación y ciclos diarios de actividad, pero casi no existen datos de las especies urbanas tropicales. Mediante recolección en el campo y aplicación de cebos en Alajuela, Costa Rica, obtuve 902 registros. Identifiqué 15 especies de hormigas urbanas, con preferencias por ciertos alimentos, y ciclos diarios afectados por disponibilidad de alimento y presencia de especies dominantes.

Palabras clave: ciclo circadiano, Glucosa, Fructuosa, Sacarosa, cronobiólogico, fotoperiodo, extirpadora.
La actividad de las hormigas varía de manera rítmica en fase con los ciclos de rotación de la Tierra y de su traslación alrededor del Sol, y esas variaciones rítmicas alcanzan los valores mínimos y máximos en distintas horas del ciclo, lo que sugiere un sistema crono-biológico interno integrado, por lo que los ritmos biológicos de periodicidad diurna o estacional pueden persistir incluso en condiciones ambientales constantes. En condiciones normales los ritmos diarios están sincronizados por las variaciones geofísicas (Aréchiga, 2004).

Estudios de campo y laboratorio han revelado que en especies como Tapinoma nigerrima y Crematogaster scutellaris la actividad de las colonias pueden variar durante el día y con las estaciones (Redolfi, Tinaut, Pascual \& Campos, 2003). Además, las hormigas dedican una mayor actividad a los recursos preferidos por calidad y cantidad (Farji-Brener, Folgarait \& Protomastro, 1992). En Camponotus sp. la actividad es mayor cuando la planta produce más néctar (Novoa, Redolfi \& Ceroni, 2005). En general, la actividad de las colonias puede variar durante el día según la especie (Redolfi et al., 2003).

En Costa Rica el alimento afecta poco la actividad en Azteca constructor, actividad que en cautiverio es mayor al medio día y en el campo varía según las condiciones ambientales (Gutiérrez-Martínez \& Acuña-Sánchez, 2013). El caso contrario se da en Linepithema dispertitum, que se activa según el alimento, evitando inflorescencias jóvenes o marchitas (Gutiérrez-Martínez, 2013).

Acá presento la lista de especies más comunes en casas de habitación en una ciudad costarricense y observaciones experimentales sobre sus patrones de actividad.

Recolecté hormigas en 19 sitios (Apéndice Digital 1) y usé cebos en marzo y abril de 2015 en una casa de habitación en Palmares de Alajuela, Costa Rica $\left(10^{\circ} 3^{\prime} 31^{\prime \prime} \mathrm{N}-84^{\circ} 25^{\prime} 09^{\prime \prime} \mathrm{W}, 1005 \mathrm{msnm}\right)$. Coloqué los cebos una hora antes de iniciar los conteos y seguí revisándolos cada hora por 24 horas (cebos líquidos: glucosa, fructuosa y sacarosa; sólidos: carne cruda de vaca).

Obtuve 704 registros en los cebos líquidos (dos fechas) y 198 en los sólidos. Las especies presentes variaron según la hora del día en ambos tipos de cebo; y cada especie mostró cierta preferencia por algún tipo de cebo (Apéndice Digital 2). Identifiqué 15 especies de hormigas urbanas, de las cuales 8 llegaron a los cebos: Camponotus atriceps, Camponotus novogranadensis, Linepithema 
angulatum, Monomorium florícola, Paratrechina longicornis, Pheidole pugnax, Pheidole punctatissima y Tapinoma melanocephalum (Apéndice Digital 3).

La actividad de las distintas especies de hormigas coincide con lo esperado por condiciones ambientales (Silva, 2010) y competencia entre especies (Hölldobler y Wilson 1990, Achury et al, 2008). La preferencia por ciertos alimentos, como soluciones azucaradas, no es sorprendente porque está bien documentada (Rico \& Moráis, 2006).

Especies comunes durante el día, como Paratrechina longicornis, son oportunistas (Hölldobler \& Wilson, 1990) y tienen la estrategia de llegar primero, pero pueden ser desplazadas por especies agresivas como Linepithema angulatum. Otras, como Monomorium floricola, incluyen en su estrategia el ser pequeñas e inconspicuas (Hölldobler \& Wilson, 1990).

Algunas especies dominan mediante la agresión (Hölldobler \& Wilson, 1990), como se observó en Tapinoma melanocephalum, Linepithema angulatum y Camponotus atriceps. En este estudio, Camponotus atriceps, bastante agresiva, desplazó a Pheidole pugnax y a Camponotus novogranadensis (ambas especies escaseaban en los cebos en la noche, cuando eran dominados por C. atriceps; ver Hölldobler \& Wilson, 1990; Achury, Chacon de Ulloa \& Arcilla, 2008).

Como las especies dominantes desplazan a las demás (Hölldobler \& Wilson, 1990), y también hay efectos de temperatura y otros factores (Gutiérrez-Martínez \& Acuña-Sánchez, 2013), recomiendo para estudios posteriores aislar las especies en laboratorio.

\section{AGRADECIMIENTOS}

Agradezco a Paul Hanson Snortun (Universidad de Costa Rica) por todo su apoyo y consejo durante el estudio y a Julián Monge-Nájera por proponer mejoras al manuscrito.

\section{REFERENCIAS}

Achury, R., Chacon de Ulloa, P., \& Arcilla, Á. M. (2008). Composición de hormigas e interacciones competitivas con Wasmannia auropunctata en fragmentos de Bosque seco Tropical. Revista Colombiana de Entomologia, 34(2), 209-215.

Aréchiga, H. (2004). Sustrato neural de los ritmos biológicos. Mensaje Bioquímico, 28, 225-250.

Farji-Brener, A. G., Folgarait, P., \& Protomastro, J. (1992). Asociación entre el arbusto Capparis retusa (Capparidaceae) y las hormigas Camponotus blandus y Acromyrmex striatus (Hymenoptera: Formicidae). Revista de Biología Tropical, 40(3), 341-344.

Gutiérrez-Martínez, P. R. (2013). Actividad de la hormiga Linepithema dispertitum (Hymenoptera: Formicidae) a lo largo del día y en diferentes estados de inflorescencia de Heliconia rostrata (Heliconiaceae). UNED Research Journal, 5(1), 57-61.

Gutiérrez-Martínez, P. R., \& Acuña-Sánchez, D. (2013). Patrones diarios de actividad de la hormiga Azteca constructor (Hymenoptera: Formicidae) y su relación con la presencia de alimento. UNED Research Journal, 5(2), 217-225.

Hölldobler, B., \& Wilson, E. O. (1990). The ants. Cambridge. Massachusetts, USA: Harvard University Press.

Novoa. S., Redolfi, I., \& Ceroni, A. (2005). Patrones de Actividad diario de la hormiga Camponotus sp. en los botones florales del cactus Neoraimondia arequipensis subs. roseiflora (Werdermann \& Backeberg) Oztolaza. Ecología Aplicada, 4(1/2), 77-81.

Redolfi, I., Tinaut, A., Pascual, F., \& Campos, M. (2003). Patrón de actividad de Tapinoma nigerrima (Nylander) y Crematogasterscutellaris (Oliver) (Hymenoptera, Formicidae) en el cultivo de olivo y en el laboratorio. Zoología Baetica, 14, 37-55.

Rico, V. G., \& Morais, H. C. (2006). Efecto de una fuente de alimentación experimental sobre una asociación HormigaHemíptero. Acta Zoológica Mexicana, 22(3), 23-28.

Silva, F. (2010). Trastornos del ritmo Diario del sueño: fisiopatología, clasificación y tratamientos. Revista Memoriza. com, 7, 1-13. 